\title{
Anti-hyperglycaemic and anti-hyperlipidaemic effects of black and red rice in streptozotocin-induced diabetic rats
}

\author{
Payungsak Tantipaiboonwong ${ }^{\mathrm{a}, *}$, Komsak Pintha ${ }^{\mathrm{a}}$, Wittaya Chaiwangyen ${ }^{\mathrm{a}}$, Teera Chewonarin ${ }^{\mathrm{b}}$, \\ Kanjana Pangjit ${ }^{c}$, Orada Chumphukam ${ }^{\mathrm{a}}$, Napapan Kangwan ${ }^{\mathrm{d}}$, Maitree Suttajit ${ }^{\mathrm{a}}$ \\ a Division of Biochemistry and Nutrition, School of Medical Sciences, University of Phayao, Phayao, \\ Thailand 56000 \\ b Department of Biochemistry, Faculty of Medicine, Chiang Mai University, Chiang Mai, Thailand 52000 \\ c College of Medicine and Public Health, Ubon Ratchathani University, Ubon Ratchathani, Thailand 34190 \\ ${ }^{d}$ Division of Physiology, School of Medical Sciences, University of Phayao, Phayao, Thailand 56000
}

*Corresponding author, e-mail: payungsak.t@gmail.com

\begin{abstract}
Increasing numbers of diabetic patients, especially those with type II, can link their condition to changes in eating habits. Black rice and red rice are not only a source of carbohydrate but also rich sources of antioxidants, especially proanthocyanidins and anthocyanins. This study determines the effect consuming a black rice extract (BRE) or a red rice extract (RRE) on streptozotocin-induced diabetic rats. Blood levels of glucose, triglycerides, and cholesterol in the diabetic group were determined and compared to that of the control group. The results demonstrate that consumption of BRE, $50 \mathrm{mg} / \mathrm{kg}$ body weight (BW), or RRE, $100 \mathrm{mg} / \mathrm{kg}$ BW, could significantly reduce the blood glucose level of the diabetic rats after eight weeks. Consumption of BRE, $100 \mathrm{mg} / \mathrm{kg}$ BW or RRE, $50 \mathrm{mg} / \mathrm{kg} \mathrm{BW}$, also clearly decreased the triglyceride level. The cholesterol level in the diabetic group fed with BRE or RRE decreased as well. Thus daily consumption of black rice, red rice, or having their extracts in food supplements may prevent the onset of diabetes.
\end{abstract}

KEYWORDS: oxidative stress, antioxidant, anthocyanins, proanthocyanidins, food supplements

\section{INTRODUCTION}

A recent report from the International Diabetes Federation showed that there have been 415 million people worldwide living with diabetes, and this number has been increasing every year. In Thailand, according to data collected through National Health Examination Surveys, the number of diabetic patients aged 15 and over has dramatically increased in the past two decades from $2 \%$ in 1991 to $7 \%$ in $2009^{1}$. The database in the year 2012 from the Bureau of Non-Communicable Diseases of Thailand also showed that 1 in every 100 people had diabetes.

Diabetes is a chronic non-communicable disease identified by an increase of fasting blood glucose as a consequence of insufficient insulin production or loss of insulin action at the target tissues. Failure to control glucose fluxes also triggers the development of hyperglycaemia and dyslipidaemia, especially an increase in triglycerides and LDL-cholesterol and a reduction in HDL-cholesterol ${ }^{2}$. More severe effects of having high blood glucose and LDL-cholesterol come after a prolonged exposure to oxidative stress. The oxidative stress condition is defined as an imbalance between free radicals and endogenous antioxidant defences that can further contribute to cell or tissue injuries associated with several diseases including diabetes ${ }^{3,4}$.

It is essential to control numbers of diabetic patients in order to reduce the costs of medical services, informal care, and indirect costs caused by morbidity and permanent disability ${ }^{1}$. Approaches geared towards preventing diabetes, such as the promotion of healthy living, are therefore beneficial. In the present study, we determine the simplest diabetes prevention method of using local food sources, particularly coloured rice. Red rice and black rice are gaining popularity as carbohydrate food sources which provide additional benefits from their nutritional enrichment and potent sources of antioxidant phytochemicals such as $\gamma$ oryzanol, tocopherols, tocotrienols, anthocyanins, and proanthocyanidins ${ }^{5-7}$. In addition to being sources of antioxidants, many studies, particularly 
of black rice, have shown a number of beneficial properties including antiglycation capacity ${ }^{5}$, antiinflammation ${ }^{8}$, as well as an ability to attenuate blood lipid profile ${ }^{9,10}$. This study is conducted to identify the effects of black rice extract (BRE) and red rice extract (RRE) consumption on the fasting blood glucose level, body weight, and lipid profiles in streptozotocin-induced diabetic rats.

\section{MATERIALS AND METHODS}

\section{Preparation of black rice crude extracts}

Black rice and red rice were obtained from a local market in Dokkhamtai, Phayao province. Dehusked rice grains were ground to a fine powder. Then $250 \mathrm{~g}$ of rice powder was soaked in 11 of $70 \%$ (v/v) ethanol that was shaken regularly $(150 \mathrm{rpm})$ for $12 \mathrm{~h}$ at room temperature. The ethanolic extracts were subsequently separated using Whatman No. 1 filter paper. The filtrates were evaporated using a vacuum rotary evaporator at $70^{\circ} \mathrm{C}$ (Buchi Rotavapor R-200). The concentrated aqueous portions were lyophilized into powders producing red and black amorphous powder, termed red rice extract (RRE) and black rice extract (BRE), respectively. They were then stored at $-20^{\circ} \mathrm{C}$ until use.

\section{Determination of total phytochemical contents}

Total phenolic content (TPC), total flavonoid content (TFC), and total anthocyanin content (TAC) of the BRE and RRE were determined as previously described by Saikia et $\mathrm{al}^{11}$. The phenolic content in sample was expressed as mg gallic acid equivalent per gram extract using the Folin-Ciocalteu assay. The flavonoid content was measured using aluminium trichloride hexahydrate colorimetric method and expressed as mg catechin equivalent per gram extract.

The estimation of TAC was performed using the pH differential method. The TAC was calculated and expressed as mg of cyanidin-3-glucoside equivalent per gram extract.

Total proanthocyanidin content (TPAC) in the BRE and RRE was evaluated using vanillin assay as described by Pintha et $\mathrm{al}^{12}$ with modifications. The rice extract or catechin standard was mixed with reagent $\mathrm{A}(1 \%(\mathrm{w} / \mathrm{v})$ vanillin in methanol) and reagent $\mathrm{B}\left(9 \mathrm{~N} \mathrm{H}_{2} \mathrm{SO}_{4}\right.$ in methanol). The reaction temperature was incubated at $30^{\circ} \mathrm{C}$ for $15 \mathrm{~min}$. The colour of the reaction mixtures was then measured at $490 \mathrm{~nm}$. The TPAC was expressed as $\mathrm{mg}$ catechin equivalent per gram extract.

\section{Quantitative analysis of phenolic compounds}

HPLC analysis for phenolic compounds in BRE and RRE was performed using a C-18 column $(250 \mathrm{~mm}$ length $\times 4.6 \mathrm{~mm}$ inner diameter, $5 \mu \mathrm{m}$ particle). BRE and RRE were injected along with standards including protocatechuic acid, catechin, chlorogenic acids, vanillic acid, hydroxybenzoic acid, coumaric acids, and ferulic acid. Then $10 \mu \mathrm{l}$ of sample was subjected to the column. The chromatographic separation was carried out at room temperature with a flow rate $1.0 \mathrm{ml} / \mathrm{min}$ of gradient. The column was eluted by gradient of two solvents: A ( $0.1 \%$ trifluoroacetic acid in water) and B (100\% methanol). The detection was performed at $280 \mathrm{~nm}$ and $325 \mathrm{~nm}$.

\section{Quantitative analysis of cyanidin-3-glucoside (C3G) and peonidin-3-glucoside (P3G) contents}

The BRE and RRE extracts $(10 \mu l)$ were analysed by an HPLC equipped with an Allure C18 (reversed phase ODS C18), $250 \mathrm{~mm} \times 4.6 \mathrm{~mm}$ diameter of particle size. Solvents used for the separation were $0.1 \%$ TFA in water and $0.1 \%$ TFA in methanol as the mobile phase $A$ and $B$, respectively. The changing the mobile phase A to mobile phase B used a linear gradient of water to methanol in $45 \mathrm{~min}$ at flow rate $1.0 \mathrm{ml} / \mathrm{min}$. The separated molecules were monitored by UV-Vis diode array detector at $520 \mathrm{~nm}$. The anthocyanin contents were calculated by HPLC peak area compared with standard calibration curve.

\section{Evaluation of vitamin $E$ analogues and $\gamma$-oryzanol contents}

The reversed phase high performance liquid chromatography (RP-HPLC) was performed to determine vitamin $\mathrm{E}$ analogues and $\gamma$-oryzanol in colour rice extracts as described by Pintha et $\mathrm{al}^{12}$. For vitamin $\mathrm{E}$ analogues determination, $10 \mu \mathrm{l}$ of extracts and standards (tocotrienols and tocopherols) were loaded into the HPLC equipped with a C30 column (250 mm length $\times 4.6 \mathrm{~mm}$ inner diameter, $5 \mu \mathrm{m}$ particle). The column was eluted with $\mathrm{MeOH}$ : water (93:7 ratio) at a flow rate of $1 \mathrm{ml} / \mathrm{min}$. The HPLC chromatograms were monitored at $292 \mathrm{~nm}$. For the detection of $\gamma$-oryzanol, Inertsil ODS-3 C18 (250 mm length $\times 4.6 \mathrm{~mm}$ inner diameter, $5 \mu \mathrm{m}$ particle) was used as a stationary phase. The $\gamma$ oryzanol were eluted using $\mathrm{MeOH}$ : acetonitrile at a ratio of 65:35 with a flow rate of $1 \mathrm{ml} / \mathrm{min}$ and was monitored at $325 \mathrm{~nm}$. The injections were performed in triplicate and the quantity of vitamin $\mathrm{E}$ analogues and $\gamma$-oryzanol contents were expressed 
as $\mu \mathrm{g}$ per gram extract.

\section{Diabetes animal model and experimental design}

Wistar albino rats (200 and $250 \mathrm{~g}$ ) were purchased from the National Laboratory Animal Centre, Mahidol University (Bangkok, Thailand). During the experiment, the rats had access to water and rat standard diet from Perfect Companion Co. Ltd, Thailand (Carbohydrate $45 \%$, protein $24 \%$, crude fat $4 \%$, crude fibre $5 \%$, moisture $10 \%$, ash $10 \%$ with sufficient essential vitamins and minerals). They were housed under a controlled temperature and humidity at $25 \pm 2{ }^{\circ} \mathrm{C}$ and $60 \pm 5 \%$, respectively, with $12 \mathrm{~h}$ of daily lighting. The rats were randomly divided into nine groups ( $n=6$ per group). Diabetic rats were induced by a single intraperitoneal injection of $75 \mathrm{mg} / \mathrm{kg}$ body weight (BW) of streptozotocin (STZ) dissolved in $100 \mathrm{mM}$ sodium citrate buffer ( $\mathrm{pH} 4.5)$. Each group received one of the following treatments. Group I, healthy control rats treated with sodium citrate buffer alone; Group II, diabetic control rats; Group III, diabetic rats treated with $10 \mathrm{mg}$ BRE/kg BW; Group IV, diabetic rats treated with $50 \mathrm{mg}$ BRE/kg BW; Group V, diabetic

Table 1 Phenolic compounds, vitamin E isoforms, and $\gamma$-oryzanol in the ethanolic extracts of black rice and red rice.

\begin{tabular}{lcc}
\hline & BRE & RRE \\
\hline Extract yield (\% by weight) & 18.7 & 12.8 \\
Hydrophilic phytochemicals & & \\
(mg/g extract) & & \\
Total phenolic content & $161.8 \pm 2.2$ & $164.7 \pm 1.2$ \\
Total flavonoid content & $96.6 \pm 9.0$ & $105 \pm 10$ \\
Protocatechuic acid & $59.5 \pm 3.3$ & $26.5 \pm 3.6$ \\
Catechin & $29.9 \pm 8.5$ & $73.7 \pm 8.5$ \\
Chlorogenic acid & $0.8 \pm 1.1$ & $20.5 \pm 5.6$ \\
Vanillic acid & $\mathrm{ND}$ & $5.66 \pm 0.64$ \\
Hydroxybenzoic acid & $403.8 \pm 8.0$ & $\mathrm{ND}$ \\
Coumaric acid & $3.85 \pm 0.20$ & $0.55 \pm 0.09$ \\
Ferulic acid & $10.4 \pm 2.3$ & $2.71 \pm 0.38$ \\
Gallic acid & $\mathrm{ND}$ & $\mathrm{ND}$ \\
Total proanthocyanidins & & \\
$\quad$ content & $25.0 \pm 2.5$ & $72.7 \pm 5.1$ \\
Total anthocyanins content & $4.87 \pm 0.33$ & $0.33 \pm 0.05$ \\
Anthocyanins ( $\mu$ g/g extract) & & \\
Cyanidin-3-O-glucoside & $72.7 \pm 2.2$ & Trace \\
Peonidin-3- $O$-glucoside & $4.52 \pm 0.06$ & Trace \\
Lipophilic phytochemicals & & \\
( $\mu$ g/g extract) & & \\
$\gamma$-tocotrienol & $15.45 \pm 0.15$ & $39.97 \pm 0.56$ \\
$\alpha$-tocotrienol & $10.24 \pm 0.37$ & $3.03 \pm 0.07$ \\
$\gamma$-tocopherol & $2.95 \pm 0.06$ & $3.20 \pm 0.06$ \\
$\alpha$-tocopherol & $5.90 \pm 0.06$ & $2.65 \pm 0.06$ \\
$\gamma$-oryzanol (mg/g extract) & $2.34 \pm 0.05$ & $0.53 \pm 0.14$ \\
\hline
\end{tabular}

Values are means $\pm \mathrm{SD}$; ND $=$ not detectable. rats treated with $100 \mathrm{mg}$ BRE/kg BW; Group VI, diabetic rats treated with $10 \mathrm{mg}$ RRE/kg BW; Group VII, diabetic rats treated with $50 \mathrm{mg} \mathrm{RRE} / \mathrm{kg} \mathrm{BW}$; and Group VIII, diabetic rats treated with $100 \mathrm{mg}$ $\mathrm{RRE} / \mathrm{kg} \mathrm{BW}$. The extracts were orally administered for a total period of eight weeks. Body weights were determined every two weeks. Fasting blood samples were drawn from the tail vein every two weeks to determine blood glucose, triglyceride, and cholesterol levels. The experiments were carried out under the approval of the Animal Ethics Committee, Faculty of Medicine, Chiang Mai University (protocol number is 27/2553).

Blood glucose (Cat\# LIBLT00026), triglycerides (Cat\# LIBLT00059), and cholesterol (Cat\# LIBLT 00034) levels were determined using an enzymatic colourimetric kit obtained from BIOTECH, Bangkok, Thailand.

\section{Statistical analysis}

Data were presented as mean $\pm \mathrm{SD}$. The statistical analysis was performed using one way ANOVA followed by an LSD post-hoc test. The mean differences at the level of $p<0.05$ were considered to be significant.

\section{RESULTS}

\section{Extraction and phytochemical contents of BRE and RRE}

The colour of the black rice fine powder extract appeared as a deep purple, while that of the red rice extract was brown red. The percentage yields by weight of the BRE and RRE were 19\% and $13 \%$, respectively. The phytochemical contents, including TPC, TFC, phenolic compounds, proanthocyanidins, anthocyanins, vitamin $\mathrm{E}$ derivatives, and $\gamma$-oryzanol, are shown in Table 1 . It is clear that the TPC and TFC contents are similar for both BRE and RRE. Interestingly, the TPAC in RRE $(72.7 \pm 5.1 \mathrm{mg} / \mathrm{g}$ extract) is 3-fold higher than that of the BRE $(25.0 \pm 2.5)$. On the other hand, TAC in $\operatorname{RRE}(0.33 \pm 0.05 \mathrm{mg} / \mathrm{g}$ extract) is lower than in BRE ( $4.87 \pm 0.33 \mathrm{mg} / \mathrm{g}$ extract). The deep purple colour in BRE represented the anthocyanins, which is mainly cyanidin-3-O-glucoside around $73 \mu \mathrm{g} / \mathrm{g}$ extract and a small amount of peonidin-3-O-glucoside was also found. Only trace amounts of anthocyanins were detected in RRE.

The HPLC analyses of BRE and RRE exhibit various phenolic compounds. Notably, hydroxybenzoic acid was found mainly in BRE but not in RRE (Table 1). The RP-HPLC was applied to identify 
Table 2 Effect of BRE and RRE on mean body weight in normal rats, STZ-induced diabetic rats and BRE/RRE treated-diabetic rats.

\begin{tabular}{lccc}
\hline Group & \multicolumn{3}{c}{ Mean body weight (g) } \\
\cline { 2 - 4 } & Week 2 & Week 4 & Week 8 \\
\hline Normal Control & 310 & 369 & 415 \\
Diabetic Control & $174^{\mathrm{a}}$ & $148^{\mathrm{a}}$ & $147^{\mathrm{a}, \mathrm{b}}$ \\
Diabetic + BRE & & & \\
$10 \mathrm{mg} / \mathrm{kg} \mathrm{BW}$ & 159 & 149 & $129^{\mathrm{b}}$ \\
$50 \mathrm{mg} / \mathrm{kg} \mathrm{BW}$ & 175 & 170 & $128^{\mathrm{b}}$ \\
$100 \mathrm{mg} / \mathrm{kg} \mathrm{BW}$ & 178 & 207 & $159^{\mathrm{b}}$ \\
$10 \mathrm{mg} / \mathrm{kg} \mathrm{BW}$ & 172 & 168 & $147^{\mathrm{b}}$ \\
$50 \mathrm{mg} / \mathrm{kg} \mathrm{BW}$ & 183 & 183 & $159^{\mathrm{b}}$ \\
$100 \mathrm{mg} / \mathrm{kg} \mathrm{BW}$ & 185 & 183 & 172 \\
\hline
\end{tabular}

${ }^{\mathrm{a}} p<0.05$ statistically significant between diabetic control and normal control

${ }^{\mathrm{b}} p<0.05$ statistically significant between BRE and RRE treatment in STZ-induced diabetic rats between weeks 2 and 8.

lipophilic compounds in the group of tocols and $\gamma$ oryzanol. The major non-polar phytochemicals in BRE and RRE were $\gamma$-tocotrienol. The amount of $\gamma$ tocotrienol in RRE was approximately 2-fold higher than in BRE, but $\gamma$-tocotrienol, $\gamma$-tocopherol, and $\gamma$-oryzanol were more than 2-fold higher in BRE compared to RRE.

\section{Effect of BRE and RRE on mean body weights}

During the 8 weeks of experiment, the rats in each group were closely monitored and all remained alive. As shown in Table 2, the mean body weights of the normal control group were significantly higher than that of the diabetic control group, whose mean body weights started to decline after the onset of the experiment. It was clearly observed that after 8 weeks, the mean body weight in the diabetic control group was significantly lower than the mean body weight at week 2 . In the experimental groups, diabetic rats were treated with a different concentration of the BRE and RRE (10, 50 , and $100 \mathrm{mg}$ extract/kg body weight). The mean body weights were then analysed at week 2,4 , and 8. It was found that the mean body weight of the diabetic rats treated with RRE $100 \mathrm{mg} / \mathrm{kg} \mathrm{BW}$ remained steady at weeks 2 and 8 and did not show any significant difference in the same group.

\section{Effect of BRE and RRE on the fasting blood glucose level}

As shown in Table 3, the mean fasting blood glucose level in the normal control group was nearly three
Table 3 Effect of BRE and RRE on fasting blood glucose levels in normal rats, STZ-induced diabetic rats and BRE/RRE-treated diabetic rats.

\begin{tabular}{lccc}
\hline \multirow{2}{*}{ Group } & \multicolumn{3}{c}{ Blood glucose levels (mg/dl) } \\
\cline { 2 - 4 } & Week 2 & Week 4 & Week 8 \\
\hline Normal Control & $299 \pm 25$ & $311 \pm 25$ & $491 \pm 76$ \\
Diabetic Control & $828 \pm 80^{\mathrm{a}}$ & $839 \pm 81^{\mathrm{a}}$ & $1205 \pm 257^{\mathrm{a}}$ \\
Diabetic + BRE & & & \\
$10 \mathrm{mg} / \mathrm{kg} \mathrm{BW}$ & $1136 \pm 46$ & $1240 \pm 175$ & $1381 \pm 85$ \\
$50 \mathrm{mg} / \mathrm{kg} \mathrm{BW}$ & $942 \pm 44$ & $1136 \pm 186$ & $1035 \pm 148^{\mathrm{b}}$ \\
$100 \mathrm{mg} / \mathrm{kg} \mathrm{BW}$ & $1008 \pm 65$ & $819 \pm 43$ & $1017 \pm 186^{\mathrm{b}}$ \\
$10 \mathrm{mg} / \mathrm{kg} \mathrm{BW}$ & $940 \pm 12$ & $1010 \pm 73$ & $1184 \pm 116$ \\
$50 \mathrm{mg} / \mathrm{kg} \mathrm{BW}$ & $914 \pm 32$ & $1030 \pm 55$ & $1101 \pm 119$ \\
$100 \mathrm{mg} / \mathrm{kg} \mathrm{BW}$ & $835 \pm 53$ & $781 \pm 33$ & $994 \pm 256^{\mathrm{b}}$ \\
\hline
\end{tabular}

Values represent mean \pm SD.

${ }^{a} p<0.05$ statistically significant between diabetic control and normal control.

${ }^{\mathrm{b}} p<0.05$ statistically significant between BRE and RRE treatment groups and diabetic control group.

times lower than that of the diabetic control group. The diabetic rats supplemented with BRE and RRE at different doses did not show any improvement in their blood glucose profile after 4 weeks of treatment. However, at the end of week 8, the blood glucose level significantly decreased in the group treated with 50 and $100 \mathrm{mg} / \mathrm{kg}$ BW of BRE and $100 \mathrm{mg} / \mathrm{kg}$ BW of RRE. The results demonstrate that long term consumption of high doses of BRE and RRE reduces blood glucose in STZ-induced diabetes.

\section{Effect of BRE and RRE on blood triglyceride level}

The levels of triglyceride in the blood of each group are presented in Table 4. The blood triglyceride levels in the diabetic group were approximately 2.5 folds higher than that of the normal control group. The BRE and RRE supplementation at 50 and $100 \mathrm{mg} / \mathrm{kg}$ BW caused a significant decrease in the triglyceride levels. These results could be observed after two weeks of treatment. It is clearly shown that a diet including colour rice, especially the black rice, could reduce the level of triglyceride.

\section{Effect of BRE and RRE on blood cholesterol level}

The blood cholesterol levels in each group are shown in Table 5. There was an approximate increase of 2-3 times the total cholesterol level in the diabetic control group after STZ-induction. The levels were significantly different from that of the normal control group. Comparison between the 
Table 4 The effect of BRE and RRE on blood triglyceride levels in normal rats, STZ-induced diabetic rats and BRE/RRE-treated diabetic rats.

\begin{tabular}{lccc}
\hline \multirow{2}{*}{ Group } & \multicolumn{3}{c}{ Blood triglyceride levels (mg/dl) } \\
\cline { 2 - 4 } & Week 2 & Week 4 & Week 8 \\
\hline Normal Control & $51 \pm 14$ & $82 \pm 11$ & $71 \pm 33$ \\
Diabetic Control & $120 \pm 33^{\mathrm{a}}$ & $179 \pm 51^{\mathrm{a}}$ & $173 \pm 38^{\mathrm{a}}$ \\
Diabetic + BRE & & & \\
$10 \mathrm{mg} / \mathrm{kg} \mathrm{BW}$ & $112 \pm 19^{\mathrm{b}}$ & $233 \pm 14$ & $216 \pm 10$ \\
$50 \mathrm{mg} / \mathrm{kg} \mathrm{BW}$ & $101 \pm 12^{\mathrm{b}}$ & $130 \pm 38^{\mathrm{b}}$ & $100 \pm 35^{\mathrm{b}}$ \\
$100 \mathrm{mg} / \mathrm{kg} \mathrm{BW}$ & $89 \pm 17^{\mathrm{b}}$ & $119 \pm 15^{\mathrm{b}}$ & $87 \pm 10^{\mathrm{b}}$ \\
$10 \mathrm{mg} / \mathrm{kg} \mathrm{BW}$ & $106 \pm 25^{\mathrm{b}}$ & $143 \pm 10^{\mathrm{b}}$ & $163 \pm 16$ \\
$50 \mathrm{mg} / \mathrm{kg} \mathrm{BW}$ & $72 \pm 8^{\mathrm{b}}$ & $115 \pm 22^{\mathrm{b}}$ & $140 \pm 45^{\mathrm{b}}$ \\
$100 \mathrm{mg} / \mathrm{kg} \mathrm{BW}$ & $75 \pm 12^{\mathrm{b}}$ & $136 \pm 19^{\mathrm{b}}$ & $134 \pm 23^{\mathrm{b}}$ \\
\hline
\end{tabular}

Values represent mean \pm SD.

${ }^{a} p<0.05$ statistically significant between diabetic control and normal control.

${ }^{\mathrm{b}} p<0.05$ statistically significant between BRE and RRE treatment groups and diabetic control group.

diabetic group and the treatment group, reveals a significant decrease of total cholesterol, observed after 4 weeks of BRE and RRE treatment at every concentration. Although the levels of total cholesterol at week 8 decreased slightly (compared among groups in the same week), the number did not show any significant difference. Thus the levels of total cholesterol did not show any improvement after treating the animals with the BRE and RRE.

\section{DISCUSSION}

Black rice and red rice have been intensively studied since they show several beneficial biological activities in vitro and in vivo, especially antioxidant and anti-inflammation properties ${ }^{5,6,8,13}$. The biological effects of BRE and RRE on their antiglycation and antioxidant capacities in vitro and in vivo are determined. In our hands, the phytochemical analysis clearly shows that BRE and RRE contain various hydrophilic and lipophilic compounds including polyphenols, flavonoids, vitamin E, and $\gamma$-oryzanol. It has been reported that proanthocyanidins and $\gamma$ tocotrienol were mainly found in red rice ${ }^{12}$. This finding is consistent with a previous study indicating that the proanthocyanidins and $\gamma$-tocotrienol are significantly higher in RRE than those of the BRE, whereas in BRE content $\alpha$-tocotrienol and $\alpha$ tocopherol are higher than those of the RRE. In addition, the anthocyanin derivative cyanidin-3-Oglucoside is mainly found in BRE, as well as a small amount of peonidin-3-O-glucoside, which is similar to other reports ${ }^{14,15}$. Our study shows that phenolic
Table 5 The effect of BRE and RRE on total cholesterol levels in normal rats, STZ-induced diabetic rats and BRE/RRE-treated diabetic rats.

\begin{tabular}{lccc}
\hline Group & \multicolumn{3}{c}{ Total cholesterol levels (mg/dl) } \\
\cline { 2 - 4 } & Week 2 & Week 4 & Week 8 \\
\hline Normal Control & $80 \pm 19$ & $66 \pm 12$ & $99 \pm 26$ \\
Diabetic Control & $117 \pm 11^{\mathrm{a}}$ & $152 \pm 31^{\mathrm{a}}$ & $327 \pm 42^{\mathrm{a}}$ \\
Diabetic + BRE & & & \\
$10 \mathrm{mg} / \mathrm{kg} \mathrm{BW}$ & $128 \pm 17$ & $94 \pm 33^{\mathrm{b}}$ & $340 \pm 22$ \\
$50 \mathrm{mg} / \mathrm{kg} \mathrm{BW}$ & $117 \pm 4$ & $101 \pm 32$ & $319 \pm 46$ \\
$100 \mathrm{mg} / \mathrm{kg} \mathrm{BW}$ & $115 \pm 9$ & $125 \pm 26^{\mathrm{b}}$ & $290 \pm 29$ \\
$10 \mathrm{mg} / \mathrm{kg} \mathrm{BW}$ & $117 \pm 21$ & $86 \pm 18^{\mathrm{b}}$ & $354 \pm 31$ \\
$50 \mathrm{mg} / \mathrm{kg} \mathrm{BW}$ & $118 \pm 14$ & $76 \pm 32^{\mathrm{b}}$ & $318 \pm 23$ \\
$100 \mathrm{mg} / \mathrm{kg} \mathrm{BW}$ & $130 \pm 12$ & $128 \pm 33^{\mathrm{b}}$ & $311 \pm 50$ \\
\hline
\end{tabular}

Values represent mean \pm SD.

${ }^{a} p<0.05$ statistically significant between diabetic control and normal control.

${ }^{\mathrm{b}} p<0.05$ statistically significant between BRE and RRE treatment groups and diabetic control group.

compounds and lipophilic compounds in BRE and RRE are the major compounds having biological activities on the free radical scavenging capacities and is consistent with other findings ${ }^{7,8,16}$.

In our study, the in vivo testing of the protective effect of BRE and RRE on diabetes rat models was performed. Wistar rats are induced with diabetes with a single injection of streptozotocin (STZ) at a dose of $75 \mathrm{mg} / \mathrm{kg}$ body weight. STZ is commonly used as a diabetogenic agent to induce irreversible damage of $\beta$-cell. STZ causes DNA alkylation and further cell necrosis by entering the pancreatic islet through glucose transporter-2. Hence STZ can lead to the inability of the $\beta$-cell to produce and secrete sufficient insulin ${ }^{17}$. We demonstrate that hyperglycaemia developed within week 2 after induction of diabetes with STZ and it remained in this state for 8 weeks to cover the whole period of this study. Three parameters were observed including the mean body weight, blood triglycerides, and cholesterol levels. The mean body weight has inverse correlation with the blood glucose levels in STZ-induced diabetic rats. This finding is in agreement with the study of Tian et al ${ }^{16}$ in that the body weight could be one of the parameters for the study of the pathophysiology of diabetes mellitus. Similar to the hyperlipidaemia, which is another characteristic of insulin-dependent diabetes ${ }^{18-20}$, the total blood triglyceride and total blood cholesterol levels also increased in diabetic rats after a single injection of STZ. 
The positive effects of BRE and RRE on the blood glucose levels have been clearly observed in the diabetic rats that were treated with BRE and RRE at $100 \mathrm{mg} / \mathrm{kg}$ body weight for 8 weeks. The blood glucose levels of both groups significantly decreased. The BRE, furthermore, exhibited a faster action on the blood glucose reduction than RRE. This could be related to the difference of phytochemical components found in BRE and RRE. Hyperglycaemia and glucose homeostasis are potentially controlled by the levels of BRE and RRE. It was reported that the insulin sensitivity can be increased as well as the up-regulation of the GLUT4 in diabetic mice models according to the effect of cyanidin-3$O$-glucoside ${ }^{21,22}$. Boue et $\mathrm{al}^{23}$ reported the increase of the level of both GLUT1 and GLUT4 gene expression. This finding could reflect the glucose uptake in adipocytes as the GLUT1 and GLUT4 were the major glucose transporters in differentiated 3T3-11 adipocytes. Furthermore, the blood glucose level could also be controlled at the digestion stage. It has been reported that the anthocyanins in black rice bran and proanthocyanidin enriched fraction of red rice bran had an inhibitory effect on $\alpha$-glucosidase and $\alpha$-amylase which subsequently disturbed starch digestion. The glucose absorption could subsequently be suppressed ${ }^{23-26}$.

It is not only the hyperglycaemia but also the hyperlipidaemia characteristic that is found in the STZ-induced diabetes animal model. The study of high dose STZ-induced DM in musk shrew showed that high plasma lipids were found in STZ-treated shrews ${ }^{19}$. This high accumulation of triglyceride in blood might suggest the relationship of insulin depletion and the function of lipoprotein lipase ${ }^{18,19}$. According to the diabetes complications, atherosclerosis, and coronary heart disease can be developed as they closely link to the hypercholesterolaemia and hypertriglyceridaemia. The BRE and RRE can significantly reduce (between 40 and 50\%) serum triglycerides and cholesterol in STZ-induced diabetic rats. Proanthocyanidins were able to inhibit pancreatic lipase activity and consequently reduce the level of plasma triglycerides ${ }^{27}$. The lipid lowering effects of anthocyanins was also in well agreement with the studies by Tsuda et $\mathrm{al}^{28}$. The improvement of hyperlipidaemia and glucose intolerance was also demonstrated in rats treated with a high fructose diet $(5 \mathrm{~g} / \mathrm{kg})$ along with anthocyaninrich extract ${ }^{9}$. It was possibly the high content of phytochemicals including phenolics, flavonoids, vitamin $\mathrm{E}$, and $\gamma$-oryzanol in BRE and RRE provide a synergistic effect contributing to the improvement of the hyperlipidaemia condition.

The HPLC analysis from our investigation has clearly shown that the BRE and RRE contain various hydrophilic and lipophilic phytochemicals. The phenolic acids are protocatechuic acid, chlorogenic acid, vanillic acid, hydroxybenzoic acid, coumaric acid, and ferulic acid. The flavonoid group consists of catechin, proanthocyanidins, and anthocyanins. Although the hydrophilic class of phytochemicals among BRE and RRE are similar, the cyanidin3-O-glucoside and peonidin-3-O-glucoside are predominant in BRE while proanthocyanidin is a rich fraction found in RRE. The major lipophilic phytochemicals including tocotrienol $(\gamma / \alpha$ isoforms) and $\gamma$-oryzanol are found in both extracts. The phytochemicals such as protocatechuic acid, chlorogenic acid, caffeic acid, and ferulic acid found in other medicinal plants have also been reported for their antidiabetic and antihyperglycaemic activities. The activities of major enzymes related to carbohydrate metabolism were observed in the STZ-induced diabetic rats. It was demonstrated that chlorogenic acid had a stimulating effect on glucose uptake in skeletal muscle through the activation of AMPK. This compound also suppressed glucose production and reduced fatty acid synthesis in hepatic cells $^{29,30}$. The lipophilic phytochemicals also played an antidiabetic role. The antidiabetic effects of vitamin $E$ and $\gamma$-oryzanol have been reported in STZ-induced diabetic rats. It had previously shown that the STZ-induced diabetic rats treated with tocotrienol-rich fraction from palm oil could potentially stabilize the level of total cholesterol, LDLcholesterol, and triglycerides ${ }^{31}$. In addition, cholesterol synthesis and fuel oxidation were increased in STZ/nicotinamide-induced diabetic rats treated with $\gamma$-tocotrienol and $\gamma$-oryzanol rich fractions of rice bran oil as a result of hyperlipidaemic and hyperinsulinemic response suppression ${ }^{32}$. HMGCoA reductase, the key enzyme in cholesterol synthesis, was primarily targeted by tocotrienols. The presence of tocotrienols and tocopherols in BRE and RRE could together exhibit protective actions on hyperlipidaemia. In conclusion, the in vivo studies revealed that BRE and RRE are potentially beneficial in lowering blood glucose level in STZ-induced rats and improving the lipid profiles. This could be the synergistic effect of lipophilic and hydrophilic phytochemicals that have antidiabetic functions in pigmented rice extracts. Daily consumption of black rice and red rice or having their extracts as food supplements may be an approach to preventing the progression of diabetes. 
Acknowledgements: The study was funded by a 2014 University of Phayao Research Grant.

\section{REFERENCES}

1. Deerochanawong C, Ferrario A (2013) Diabetes management in Thailand: a literature review of the burden, costs, and outcomes. Glob Health 9, 11.

2. Eckel RH, Grundy SM, Zimmet PZ (2005) The metabolic syndrome. Lancet 365, 1415-28.

3. Coppey LJ, Gellett JS, Davidson EP, Dunlap JA, Lund DD, Yorek MA (2001) Effect of antioxidant treatment of streptozotocin-induced diabetic rats on endoneurial blood flow, motor nerve conduction velocity, and vascular reactivity of epineurial arterioles of the sciatic nerve. Diabetes 50, 1927-37.

4. Maritim AC, Sanders RA, Watkins JB III (2003) Diabetes, oxidative stress, and antioxidants: a review. $J$ Biochem Mol Toxicol 17, 24-38.

5. Daiponmak W, Senakun C, Siriamornpun S (2014) Antiglycation capacity and antioxidant activities of different pigmented Thai rice. Int J Food Sci Tech 49, 1805-10.

6. Laokuldilok T, Shoemaker CF, Jongkaewwattana S, Tulyathan V (2011) Antioxidants and antioxidant activity of several pigmented rice brans. J Agr Food Chem 59, 193-9.

7. Tanaka J, Nakanishi T, Ogawa K, Tsuruma K, Shimazawa M, Shimoda H, et al (2011) Purple rice extract and anthocyanidins of the constituents protect against light-induced retinal damage in vitro and in vivo. J Agr Food Chem 59, 528-36.

8. Choi SP, Kim SP, Kang MY, Nam SH, Friedman M (2010) Protective effects of black rice bran against chemically-induced inflammation of mouse skin. J Agr Food Chem 58, 10007-15.

9. Guo H, Ling W, Wang Q, Liu C, Hu Y, Xia M, et al (2007) Effect of anthocyanin-rich extract from black rice (Oryza sativa L. indica) on hyperlipidemia and insulin resistance in fructose-fed rats. Plant Foods Hum Nutr 62, 1-6.

10. Jang HH, Park MY, Kim HW, Lee YM, Hwang KA, Park $\mathrm{JH}$, et al (2012) Black rice (Oryza sativa L.) extract attenuates hepatic steatosis in C57BL/6 J mice fed a high-fat diet via fatty acid oxidation. Nutr Metabol 9, 27.

11. Saikia S, Himjyoti D, Daizi S, Charu LM (2012) Quality characterization and estimination of phytochemical content capacity of aromatic pigmented and non pigmented rice varieties. Food Res Int 46, 334-40.

12. Pintha K, Yodkeeree S, Pitchakarn P, Limtrakul P (2014) Anti-invasive activity against cancer cells of phytochemicals in red jasmine rice (Oryza sativa L.). Asian Pac $J$ Canc Prev 15, 4601-7.

13. Chen MH, Choi SH, Kozukue N, Kim HJ, Friedman M (2012) Growth-inhibitory effects of pigmented rice bran extracts and three red bran fractions against human cancer cells: relationships with composition and antioxidative activities. J Agr Food Chem 60, 9151-61.

14. Ichikawa $\mathrm{H}$, Ichiyanagi $\mathrm{T}, \mathrm{Xu} \mathrm{B}$, Yoshii $\mathrm{Y}$, Nakajima M, Konishi T (2001) Antioxidant activity of anthocyanin extract from purple black rice. J Med Food 4, 211-8.

15. Sompong R, Siebenhandl-Ehn S, Linsberger-Martin G, Berghofer E (2011) Physicochemical and antioxidative properties of red and black rice varieties from Thailand, China and Sri Lanka. Food Chem 124, 132-40.

16. Tian HL, Wei LS, Xu ZX, Zhao RT, Jin DL, Gao JS (2010) Correlations between blood glucose level and diabetes signs in streptozotocin-induced diabetic mice. Global J Pharmacol 4, 111-6.

17. Elsner M, Guldbakke B, Tiedge M, Munday R, Lenzen S (2000) Relative importance of transport and alkylation for pancreatic beta-cell toxicity of streptozotocin. Diabetologia 43, 1528-33.

18. Abbate SL, Brunzell JD (1990) Pathophysiology of hyperlipidemia in diabetes mellitus. $J$ Cardiovasc Pharmacol 16, Suppl 9, S1-7.

19. Ohno T, Horio F, Tanaka S, Terada M, Namikawa T, Kitoh J (2000) Fatty liver and hyperlipidemia in IDDM (insulin-dependent diabetes mellitus) of streptozotocin-treated shrews. Life Sci 66, 125-31.

20. Islam MS, Loots DT (2009) Experimental rodent models of type 2 diabetes: a review. Meth Find Exp Clin Pharmacol 31, 249-61.

21. Sasaki R, Nishimura N, Hoshino H, Isa Y, Kadowaki M, Ichi T, et al (2007) Cyanidin 3-glucoside ameliorates hyperglycemia and insulin sensitivity due to downregulation of retinol binding protein 4 expression in diabetic mice. Biochem Pharmacol 74, 1619-27.

22. Apichai S, Pongchaidecha A, Kaeapai W, Jitprawet N, Lailerd N (2012) Beneficial effects of Thai purple sticky rice supplement in streptozotocin induced diabetic rats. CMU J Nat Sci 11, 371-81.

23. Boue SM, Daigle KW, Chen MH, Cao H, Heiman ML (2016) Antidiabetic potential of purple and red rice (Oryza sativa L.) bran extracts. J Agr Food Chem 64, 5345-53.

24. Shimoda H, Aitani M, Tanak J, Hitoe S (2015) Purple rice extract exhibits preventive activities on experimental diabetes models and human subjects. $J$ Rice Res 3, 137.

25. Akkarachiyasit S, Charoenlertkul P, Yibchok-Anun S, Adisakwattana S (2010) Inhibitory activities of cyanidin and its glycosides and synergistic effect with acarbose against intestinal $\alpha$-glucosidase and pancreatic $\alpha$-amylase. Int J Mol Sci 11, 3387-96.

26. Premakumara GAS, Abeysekera WKSM, Ratnasooriya WD, Chandrasekharan NV, Bentota AP (2013) Antioxidant, anti-amylase and anti-glycation potential of brans of some Sri Lankan traditional and 
improved rice (Oryza sativa L.) varieties. $J$ Cereal Sci 58, 451-6.

27. Moreno DA, Ilic N, Poulev A, Brasaemle DL, Fried SK, Raskin I (2003) Inhibitory effects of grape seed extract on lipases. Nutrition 19, 876-9.

28. Tsuda $\mathrm{T}$, Horio $\mathrm{F}$, Uchida $\mathrm{K}$, Aoki $\mathrm{H}$, Osawa $\mathrm{T}$ (2003) Dietary cyanidin 3-O- $\beta$-D-glucoside-rich purple corn color prevents obesity and ameliorates hyperglycemia in mice. $J$ Nutr 133, 2125-30.

29. Meng S, Cao J, Feng Q, Peng J, Hu Y (2013) Roles of chlorogenic acid on regulating glucose and lipids metabolism: a review. Evid Based Complement Altern Med 2013, ID 297154.

30. Ong KW, Hsu A, Tan BK (2012) Chlorogenic acid stimulates glucose transport in skeletal muscle via AMPK activation: a contributor to the beneficial effects of coffee on diabetes. PLOS ONE 7, e32718.

31. Budin SB, Othman F, Louis SR, Bakar MA, Das S, Mohamed J (2009) The effects of palm oil tocotrienolrich fraction supplementation on biochemical parameters, oxidative stress and the vascular wall of streptozotocin-induced diabetic rats. Clinics 64, 235-44.

32. Cheng HH, Ma CY, Chou TW, Chen YY, Lai MH (2010) Gamma-oryzanol ameliorates insulin resistance and hyperlipidemia in rats with streptozotocin/nicotinamide-induced type 2 diabetes. Int $J$ Vitam Nutr Res 80, 45-53. 